\title{
Exploring Casual Exergames With Kids Using Wheelchairs
}

\author{
Kieran Hicks \\ University of Lincoln \\ Brayford Pool \\ Lincoln LN6 7TS, UK \\ khicks@lincoln.ac.uk

\section{Kathrin Gerling} \\ University of Lincoln \\ Brayford Pool \\ Lincoln LN6 7TS, UK \\ kgerling@lincoln.ac.uk
}

Permission to make digital or hard copies of part or all of this work for personal or classroom use is granted without fee provided that copies are not made or distributed for profit or commercial advantage and that copies bear this notice and the full citation on the first page. Copyrights for third-party components of this work must be honored. For all other uses, contact the O wner/Author.

Copyright is held by the owner/author(s).

CHI PLAY 2015, O ctober 03-07, 2015, London, U nited Kingdom A CM 978-1-4503-3466-2/15/10.

http://dx.doi.org/1 0.1145/2793107.2810304

\begin{abstract}
Physical activity (PA) is important for health and wellbeing, but often PA is inaccessible for children using wheelchairs. In this work, we explore the potential of casual exergames to provide opportunities for physically active play. We apply existing wheelchaircontrolled video games to explore children's and parents' perceptions of these games. Feedback shows that children and parents feel that casual exergames could be a valuable opportunity of offering wheelchairaccessible play, however, refinement of game concepts particularly regarding challenge and physical effort required to play is necessary. By integrating these findings into games for young people using wheelchairs in the future, we hope to provide opportunities for accessible and physically challenging play.
\end{abstract}

\section{Author Keywords}

Game accessibility, game design, casual exergames.

\section{ACM Classification Keywords}

K.8.0 [Personal Computing]: General - Games.

\section{Introduction}

Getting sufficient levels of physical activity is essential to health and well-being. Recent research has demonstrated the importance of regular activity throughout the day in addition to more intense 
workouts, suggesting that sedentary behavior (e.g., long periods of sitting among students or office workers) has negative health implications distinctly different from those that result from a lack of intense physical activity [10].

In this context, people using wheelchairs are at a higher risk of experiencing negative consequences of a lack of physical activity as access barriers are higher [9], and particularly among children with disabilities, opportunities for physically stimulating play are limited [1], leading to higher-than-average levels of sedentary behavior throughout the day. Casual exergames movement-based games that are intuitive to use and can be played in short chunks of time [1] - have been demonstrated to be an effective means of breaking up periods of sedentary behavior among non-disabled children [1], [5], however, their accessibility for kids using wheelchairs is limited.

In this paper, we explore the potential of wheelchaircontrolled casual exergames to make the benefits of physically challenging games available to kids using wheelchairs. We selected two games from previous game development projects [2] that looked into wheelchair-controlled movement-based games, Skiing Mountain a wheelchair-controlled skiing game and Collector of Light, a fantasy-themed infinite runner in which wheelchair movement controls an elk. Both games fit the definition of casual exergames in the sense that they provide easily accessible gameplay that could be broken up into short chunks. We worked with Go Kids Go, a charity that provides wheelchair skills training for young people, to integrate the games into skills training sessions to examine perceptions that children and parents had of casual exergame play. Five children and four parents participated in this initial step of our research, and results suggest that participants consider casual exergames a valuable opportunity for wheelchair-accessible, physically stimulating play, however, further refinement of game concepts particularly regarding challenge and effort required to play is necessary.

Based on these findings, we outline opportunities for future research in the development of games enabling young people using wheelchairs to participate in PA. By creating casual exergames specifically addressing young people using wheelchairs, we hope to provide opportunities for accessible and physically challenging play that is available throughout the day.

\section{Casual Exergames for Children Using Wheelchairs}

This section outlines the design rationale behind the two games included in this study, which both offer casual play integrating simple wheelchair movements.

Movement-Based Games for Players With Disabilities Our work builds on existing research into movementbased games for people with mobility disabilities, and projects exploring wheelchair-controlled play.

A study by Hernandez et al. [4] on creating exergames for children with cerebral palsy found that children found slow-paced games boring, and concluded that it was possible to have fast action games providing the gameplay developed around them. Regarding wheelchair-controlled play, O'Conor et al. [8] found that participants enjoyed being able to use their personal wheelchair as input device, and that they experienced flow when engaging with a racing game, 


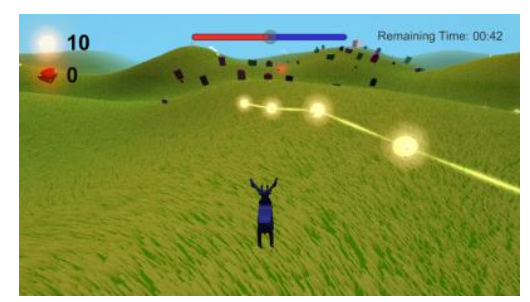

Figure 1. Collector of Light, an infinite runner in which the player controls an elk. Wheelchair turns to the left and right control direction, while moving back and forth controls speed.

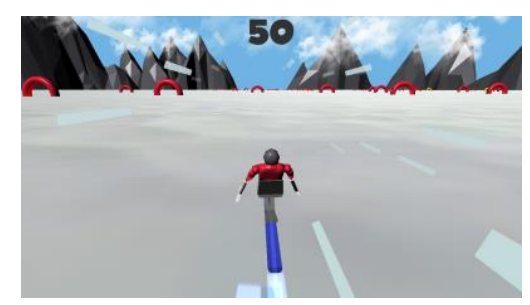

Figure 2. Skiing Mountain, a skiing game featuring three different game modes in which wheelchair movement controls the skier. suggesting that this kind of genre has potential to draw players in as wheelchair movement and in-game action map closely. In our games, we try to reflect these aspects through the choice of genre - infinite runners which give the player the feeling of speed throughout play. Additionally, whilst designing player controls, results from previous work were considered, e.g., the time it would take for users to perform wheelchair movement [3]. This also extended to technical limitations, where gameplay must not require the player to turn more than 45 degrees. Gameplay should also not require to move out of range of the Kinect sensor e.g. asking the player to move forward several times without moving backwards [3].

\section{Development Process}

Games included in our research were developed following two different approaches [2]. For the first game, Skiing Mountain, focus groups were conducted with young people with complex needs to establish a co-design process and identify suitable game themes along with basic game mechanics. The second game, Collector of Light, was designed and developed by a group of final-year game design students over the course of 12 weeks where they were first instructed to created personas and then design games in groups using these personas as the player. Both games were created using Unity 5, and implement wheelchair input using the KINECT ${ }^{\text {Wheels }}$ toolkit [3].

\section{GAme 1: SKIING Mo UnTAIN}

Skiing Mountain is a prototype skiing game comprised of three different modes that vary in duration to suit different lengths of play sessions.
Interaction. The player controls a Skier avatar using an assistive skiing device by moving the wheelchair forward, backward, and by turning to the sides.

Gameplay. The first mode is Speed slope (Figure 2), where the player has 60 seconds to travel as far down the mountain as they can. The avatar automatically goes forward down the mountain while the player has control over turning left and right which is done by physically turning the wheel chair in the respective direction. The second mode is Slalom Gates. In this mode, the player controls the same avatar as the previous mode but must try to go in-between the different slalom gates whilst traversing the mountain. The course of this mode was designed to last around two minutes to provide a slightly longer and more structured play session. The final mode of the game is Free Ride. In this mode the player once again controls the same avatar using the same control scheme. There are no goals in this mode and instead the player rides down the mountain at their own pace. This mode was designed to last three to four minutes, and to provide a non-objective based mode.

GAME 2: CO LLECTOR OF LIGHT

The second game used during the study was Collector of Light, an infinite runner in which the player controls an elk in a procedurally generated world.

Interaction. The player can steer the elk by physically moving the wheelchair left or right, while moving back and forth controls speed. A bar at the top of the screen provides direct feed back to the player to show how much the elk will turn. 


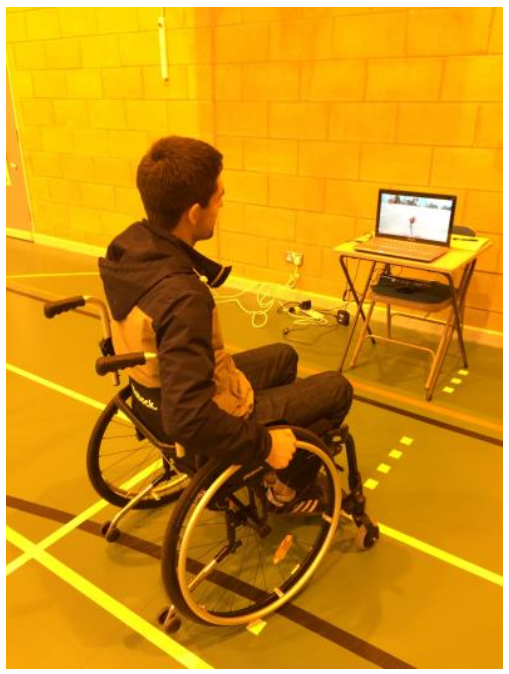

Figure 3. One of the researchers demonstrating the system setup used by participants.
Gameplay. The player steers the elk with the goal of collecting as many light orbs as possible in a 60 second session. There are also obstacles that spawn in the world that the player must strive to avoid as crashing into them causes the player to lose a portion of their collected light orbs. The world is populated with powerups to give the player bonus powers such as increasing the pickup radius of globes, or making the player immune to crashing into obstacles for a period of time.

Both games fit the definition of casual exergames in the sense that they provide easily accessible, intuitive gameplay that can be split up into short amounts of time [1], therefore making it easier to integrate gaming into daily routines. In the next step of our research, we apply these games to gain insights into perspectives of children using wheelchairs and their parents on wheelchair-controlled casual exergames to outline basic design opportunities for such games.

\section{Exploring Player Perceptions of Wheelchair- Controlled Casual Exergames}

We explored the potential of casual exergames for kids using wheelchairs through an initial user study carried out at two wheelchair skills training workshops hosted by Go Kids Go in Hull and Sheffield in the UK. In our study, we were particularly interested in (1) children's perceptions of our games, (2) the way they interacted with them, and (3) the perspectives parents had on play and physical activity.

Participants and Procedure

Five children (average age nine, two female) and four parents participated in the study. All children used manual wheelchairs, and played sedentary video games on a regular basis. At the beginning, all participants were informed about the research. Parents gave written consent on their own behalf and for their children to participate in our study. Additionally, we followed an oral assent protocol with all children during which we explained the research project and asked participants to state whether they agreed to participate in the study. Following this step, we asked children about physical activity routines and gaming preferences, and invited them to try both games for about ten minutes of play (Figure 3 ). While children engaged with the games, we made observations and asked parents about their impressions and their children's activity patterns. Afterwards, we carried out short post-play interviews with the children about their experience with our games. This research was approved by the University of Lincoln College of Science ethics board, and reviewed by Go Kids Go.

\section{Results}

Results were analyzed with a focus on the three previously identified areas of interest. We followed recommendations for qualitative research by Marshall and Rossman [6]

Children's perceptions of the games. Generally, participants were enthusiastic about the idea of wheelchair-controlled games, stating that "it feels like you're involved, very physical", that "it's fun because it [the avatar] follows your movement" (P2, child), and that they "really enjoyed it" (P2, child). Responses to themes and game mechanics were mixed. While some participants appreciated the nature of infinite runners, other participants pointed out that they generally played on different platforms and preferred different genres, with favourite games such as Minecraft or Animal Crossing. 
Interaction with the games. Regarding player interaction with the games, wheelchair input was suitable for all participants, and tracking was stable despite individual differences in body size or posture. Observations showed that all participants had an intuitive understanding of game controls. Regarding game controls, some participants were happy with the current implementation, outlining that the game "is really fun because you're in control, because it's very exact" ( $\mathrm{P} 1$, child) however, some participants experienced difficulties adapting to the sensitivity of controls, stating that "it was a bit difficult to steer" (P3, child), suggesting that it might be helpful to be able to make individual adjustments depending on the level of motor control that players have when navigating their wheelchair.

Parents' perspectives on play and physical activity. Parents were interested in the idea of casual exergames as it would open up opportunities for their children to engage in physical activity throughout the day. Although all children were physically active, participating in sports ranging from wheelchair basketball to swimming and dance, parents pointed out that access to physically stimulating playful activity remained difficult. This was echoed by children's statements, with one participant commenting that he "can't join in with most [playground] games", and he "usually just finds somebody to talk to" (P4, child). Specifically, parents were interested in games that could facilitate wheelchair skills as training sessions were not available on a regular basis, and games that could contribute to general fitness. In this context, one parent suggested that "it would be good to have upperbody movement, too" (P6, parent).

\section{Discussion and Future Directions}

This paper presents an enquiry into design opportunities and limitations when developing casual exergames for children using wheelchairs. Two games were tested in an exploratory setting, investigating the perceptions that children and parents had on play. Results show that feedback was generally positive, but that the concept of wheelchair-controlled casual exergames needs further refinement. In this section, we discuss design opportunities beyond basic adaptations to improve playability that we aim to explore building on this work.

\section{Design Opportunities}

The main area of interest in the development of wheelchair-controlled casual exergames that we wish to explore in the future is concerned with exploring more physically challenging wheelchair-controlled play.

While both games included in this study integrated basic wheelchair movement, we plan to study levels of exertion that can be achieved through wheelchaircontrolled casual exergames. This is particularly interesting as parents in the study showed interest in having the games that were tested deployed in a more permanent setting as only few home-based physically stimulating playful activities are available. Such games would have to be designed in a way that requires the player to move the wheelchair more actively, possibly also including upper body movement.

Additionally, building on children's feedback further work is necessary to ensure that the player both finds the activity of playing both physically exercising but also enjoyable is a challenge as participants voiced a range of gaming preferences. In the future, this could 
be mitigated by providing an array of mini games that span the more popular genres.

\section{Conclusion}

Preliminary results from our research into wheelchaircontrolled casual exergames demonstrate potential of such games to provide opportunities for PA for young people using wheelchairs.

Generally, wheelchair-controlled casual exergames offer an opportunity to re-think the role of exergames in the lives of players. While previous research usually focused on games as a means of motivating players to be physically active (e.g., [5], [8]), creating games for players with mobility disabilities comes with a shift in perspective: rather than motivating players to engage in an activity that seems unappealing, many players using wheelchairs expressed the desire to be more physically active and were already motivated to exercise, but lacked opportunities to engage in PA. To this end, the design of wheelchair-controlled casual exergames can adopt a more positive perspective on players, using the game as a vehicle to enable players to participate in an activity that they perceive as appealing, rather than coercing them into exercising by disguising PA as an interactive game.

\section{Acknowledgments}

We would like to thank Roy Wild and Emily Ellington at Go Kids Go and all study participants for their support.

\section{References}

[1] Gao, Y., Gerling, K., Mandryk, R., and Stanley, K. Decreasing Sedentary Behaviours in Pre-adolescents using Casual Exergames at School. Proc. of CHI PLAY 2014, ACM (2014), 97-106.
[2] Gerling, K., Linehan, C., Kirman, B., Kalyn, M., Evans, A., and Hicks, K. 2015. Creating WheelchairControlled Video Games: Challenges and Opportunities when Involving Young People with Mobility Impairments and Game Design Experts.

[3] Gerling, K., Mandryk, R., Miller, M., Kalyn, M., Birk, M., Smeddinck, J. Designing wheelchair-based movement games. TACCESS 6, 2 (2015), Article 6.

[4] Hernandez, A., Ye, Z., Graham, N., Fehlings, D., Switzer, L. (2013). Designing Action-based Exergames for Children with Cerebral Palsy. In: Proc. Of CHI 2013, ACM

[5] Mandryk, R., Gerling, K., and Stanley, K. Designing Games to Discourage Sedentary Behaviour. A. Nijholt

(Ed.): Playful User Interfaces. Springer (2014).

[6] Marshall, C., and Rossman, G.B. 2011. Designing Qualitative Research. Sage Publishers: Thousand Oaks, CA, USA

[7] Missiuna, C., and Pollock, N. Play Deprivation in Children With Physical Disabilities: The Role of the Occupational Therapist in Preventing Secondary Disability. The American Journal of Occupational Therapy 45, 10 (1991), 882-888.

[8] O'Connor, J., Fitzgerald, S., Boninger, M. Evaluation of Manual Wheelchair Interface to Computer Games. Neurorehabilitation and Neural Repair $14,(2000), 21-31$

[9] Rimmer, J., Riley, B., Wang, E., Rauworth, A., and Jurkowski, J. Physical activity participation among persons with disabilities: Barriers and facilitators. American Journal of Preventive Medicine 26, 5 (2004), 419-425.

[10] Tremblay, M., Colley, R., Saunders, T., Healy, G., and Owen, N. Physiological and health implications of a sedentary lifestyle. Applied Physiology, Nutrition, and Metabolism 35, 6 (2010), 725-740. 